\title{
The wide-field spatio-spectral interferometer: system overview, data synthesis and analysis
}

Roser Juanola-Parramon, Alex lacchetta, David T. Leisawitz, Matthew R. Bolcar, Stephen F. Maher, et al.

Roser Juanola-Parramon, Alex lacchetta, David T. Leisawitz, Matthew R. Bolcar, Stephen F. Maher, Stephen A. Rinehart, "The wide-field spatiospectral interferometer: system overview, data synthesis and analysis," Proc. SPIE 10701, Optical and Infrared Interferometry and Imaging VI, 107011K (9 July 2018); doi: 10.1117/12.2314162

Event: SPIE Astronomical Telescopes + Instrumentation, 2018, Austin, Texas, United States 


\title{
The wide-field spatio-spectral interferometer: system overview, data synthesis and analysis
}

\author{
Roser Juanola-Parramon ${ }^{\mathrm{a}}$, Alex Iacchetta ${ }^{\mathrm{b}}$, David T. Leisawitz ${ }^{\mathrm{a}}$, Matthew R. Bolcar ${ }^{\mathrm{a}}$, Stephen \\ F. Maher ${ }^{\mathrm{a}}$, and Stephen A. Rinehart ${ }^{\mathrm{a}}$ \\ ${ }^{a}$ NASA Goddard Space Flight Center, 8800 Greenbelt Rd, Greenbelt MD, USA \\ ${ }^{\mathrm{b}}$ Institute of Optics - University of Rochester, 275 Hutchison Rd, Rochester NY, USA
}

\begin{abstract}
The Wide-field Imaging Interferometry Testbed (WIIT) is a double Fourier (DF) interferometer operating at optical wavelengths, and provides data that are highly representative of those from a space-based far-infrared interferometer like SPIRIT. We have used the testbed to observe both geometrically simple and astronomically representative test scenes. Here we present an overview of the astronomical importance of high angular resolution at the far infrared, followed by the description of the optical set-up of WIIT, including the source simulator CHIP (Calibrated Hyperspectral Image Projector). We describe our synthesis algorithms used in the reconstruction of the input test scenes via a simulation of the most recent measurements. The updated algorithms, which include instruments artifacts that allow the synthesis of DF experimental data, are presented and the most recent results analyzed.
\end{abstract}

Keywords: double Fourier modulation, spectro-spatial interferometry, spectroscopy, interferometry, space instrumentation

\section{INTRODUCTION}

Lacking sub-arcsecond angular resolution in the far-infrared $(\sim 25-400 \mu m)$, astrophysicists are presently unable to test competing models for the formation of planetary systems and the delivery of water to potentially habitable planets, and are missing information that will be key to understanding the formation and evolution of galaxies. With the Herschel Space Observatory, at $3.5 \mathrm{~m}$ the largest space telescope flown to date, even the nearest protoplanetary systems were unresolved, and light from multiple distant galaxies was blended in each resolution element. Aided by high-resolution spectroscopy and models, it is possible to interpret Herschel observations of these objects, though not definitively. The objects of interest will have to be well resolved spatially to break model degeneracies and understand planet, star, and galaxy formation more deeply, and this requires sub-arcsecond angular resolution.

A single-aperture telescope operating at $100 \mu \mathrm{m}$ would need to have a diameter of $25 \mathrm{~m}$ just to provide 1 arcsecond resolution. A cryogenic space telescope of this size may be unaffordable with current budgets for space science, and certainly isn’t necessary to make tremendous scientific progress.

In the NASA Astrophysics Roadmap, Enduring Quests, Daring Visions, ${ }^{1}$ "several of the notional missions ... rely on interferometry to answer key science questions, from radio to X-rays." Accordingly, the Roadmap notes that "technology maturation of interferometric techniques is thus highly relevant to realizing the science vision." Further, the Roadmap makes this practical observation: "Interferometry has historically progressed from longer

E-mail: roser.juanola@nasa.gov

Optical and Infrared Interferometry and Imaging VI, edited by Michelle J. Creech-Eakman,

Peter G. Tuthill, Antoine Mérand, Proc. of SPIE Vol. 10701, 107011K · (c) 2018 SPIE

CCC code: $0277-786 \mathrm{X} / 18 / \$ 18 \cdot$ doi: $10.1117 / 12.2314162$

Proc. of SPIE Vol. 10701 107011K-1 
wavelengths, where technological challenges are less extreme, to shorter wavelengths." The overarching objective of our research program is to determine if the wide-field spatio-spectral interferometry technique can fulfill the community's science-driven measurement requirements at far-infrared wavelengths.

Spatio-spectral interferometry, also called double Fourier modulation, is the application of Fourier transform spectroscopy to aperture synthesis interferometry. This technique was proposed for the near IR regime by Itoh and Ohtsuka ${ }^{2}$ (1986) with a single-pupil interferometric approach, and by Mariotti and Ridgway ${ }^{3}$ (1988) with multi-pupil interferometry for high spatial resolution. Leisawitz et al. ${ }^{4}$ (2003) proposed an extension of the fieldof-view (FOV) by using a focal plane detector array. This technique, called wide-field imaging interferometry, is presented here for optical wavelengths, as direct detector arrays in the far infrared are still expensive and a similar approach is not straightforward. Ohta et al. ${ }^{5}$ (2006) theoretically proposed to apply a Martin-Pupletttype Fourier transform spectrometer to the aperture synthesis system in millimeter and submillimeter waves. They succeeded in proving that this system is capable of performing broadband imaging observations ${ }^{6}(2007)$. Also a laboratory prototype spectral-spatial interferometer located at Cardiff University has demonstrated the feasibility of the double Fourier technique at far infrared (FIR) wavelengths $(0.15-1 \mathrm{THz})$ by Grainger et al. ${ }^{7}$ (2012) with a single pixel detector.

Double Fourier modulation (DFM) is based on the combination of spatial interferometry and Fourier transform spectroscopy simultaneously. A Fourier transform spectrograph is based on the Michelson interferometer, where the light of a source is divided and recombined while delaying one of the optical paths to generate an interferogram. To combine this technique with spatial interferometry, instead of dividing the light from a single telescope one uses two apertures and recombines the incoming light from these two apertures.
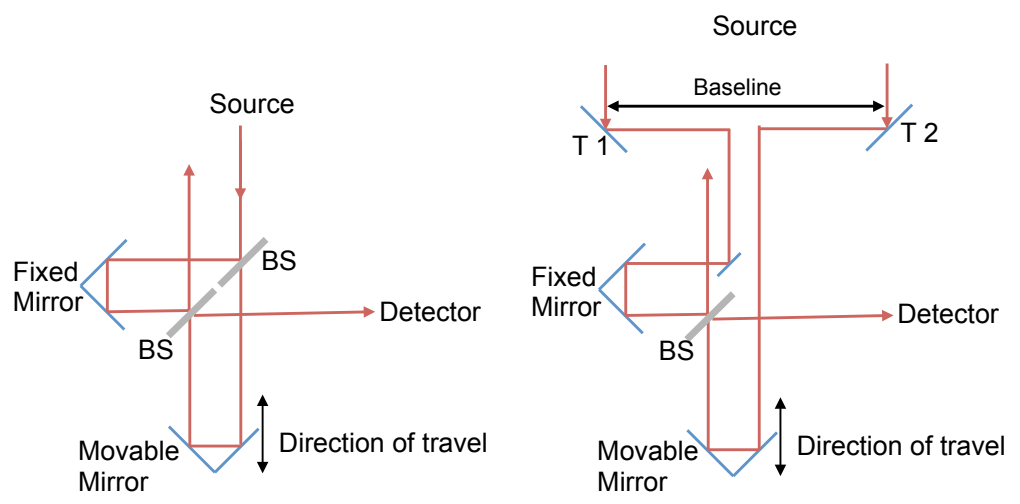

Figure 1. Schematic of a classical FTS (left) and a spectro-spatial interferometer (right). For the FTS, the incoming light is divided in the first beam splitter and half of it is delayed with the movable mirror before arriving to the second beam splitter where interference occurs. The spectro-spatial interferometer receives light from two telescopes (T1 and T2), and the light travelling from $\mathrm{T} 2$ is delayed at the movable mirror before interfering with the light travelling from T1 at the beam splitter, where interference occurs.

Fig. 1 shows a classical FTS (left) and a spectro-spatial interferometer (right). In the FTS the incoming light is divided at the beamsplitter (BS) and with the movable mirror an optical path difference is introduced. In the spectro-spatial interferometer, by using two telescopes an extra path difference due to the baseline is introduced when the source is not on the line of sight perpendicular to the baseline. When using the two telescope interferometer, due to the division of the spatial wavefront outside the instrument, one has to take into account all the phase changes of the optical elements. ${ }^{3}$ Considering that all mirrors introduce a $\pi$ phase delay and that the beam splitter is ideal and symmetric, the phase delay between the transmitted and reflected beam 
is $\pi / 2$ and the detector measures sine interferograms due to the extra $\pi / 2$ phase shift. Because this is the result of the addition of the beam splitter phase $\phi_{B S}$, throughout this paper this value is left as a variable in order to account for beam splitter non-symmetries.

\section{EXPERIMENTAL APPROACH}

The Wide-field Imaging Interferometry Testbed ${ }^{4}$ (WIIT) is located at NASA's Goddard Space Flight Center, specifically at the Goddard Advanced Interferometry and Metrology Laboratory (AIM Lab). The AIM Lab is a world-class facility, which was developed with the requirements of this particular experiment in mind. A combination of thermal stability, acoustic isolation, passive and active vibration control, and gentle, laminar air flow in a clean room allow the data obtained with WIIT in the AIM Lab to be practically free of environmentally induced errors.

The design and assembly of the testbed took place over a two-year period beginning in May 1999, and culminated in detection of the first white light fringes in 2001, but major improvements have been implemented since. $^{8}$ It is designed to develop and validate the dual-Michelson interferometric technique. By combining Michelson stellar interferometry with Fourier-transform spectroscopy and using a detector array instead of a single-pixel detector, the WIIT is capable of measuring high-resolution spatial-spectral data over a large FOV, which will be detailed in the next section.

\subsection{Optical layout}

WIIT is a 1:150 functional scale model of the Space Infrared Interferometric Telescope (SPIRIT), ${ }^{9}$ and is designed to observe complex scenes representative of far-IR astronomical fields. It provides full uv-plane coverage and a wide FOV, operating at visible rather than far-IR wavelengths for several practical reasons: ${ }^{10}$ (a) the optical apertures and the delay line are scaled down from those intended for a far-IR instrument in proportion to the wavelength; (b) a CCD detector with sensitivity limited by photon noise (as expected in a cold far-IR space interferometer) was commercially available and affordable; (c) many other parts, such as translation and rotation stages, were available off-the shelf and inexpensive; and (d) a commercial optical metrology system accurate to $10 \mathrm{~nm}$ was affordable.

The novel aspect of WIIT is the use of a detector array (a CCD camera) after the beam splitter instead of the single-pixel detector used in a traditional Michelson interferometer (as in the Cardiff University Testbed). Each pixel on the detector records light arriving from different parts of the sky (test scene). As the delay line is scanned, each pixel records an interferogram unique to the field angle corresponding to the pixel. By using observations at a wide range of $u v$-positions, obtained by rotating the source and increasing/decreasing the interferometric baseline length, reconstructed images can be produced for each pixel; these images can then be mosaiced together, producing a wide field-of-view image with the full interferometric angular resolution. In reality, a more sophisticated algorithm is used to generate a spatial-spectral datacube covering the wide field of view. ${ }^{11,12}$ By providing a long delay line scan, spectral information for the observed sources is obtained. To obtain a spectral resolution $R$ requires an optical path delay (OPD) scan range of $\delta=R \lambda$.

\subsection{Extension of the FOV}

To understand how WIIT images a wide FOV next consider a source located at an angle $\theta$ relative to the optical axis, as illustrated in Figure 2. If the angular offset is in a direction parallel to the baseline vector established 


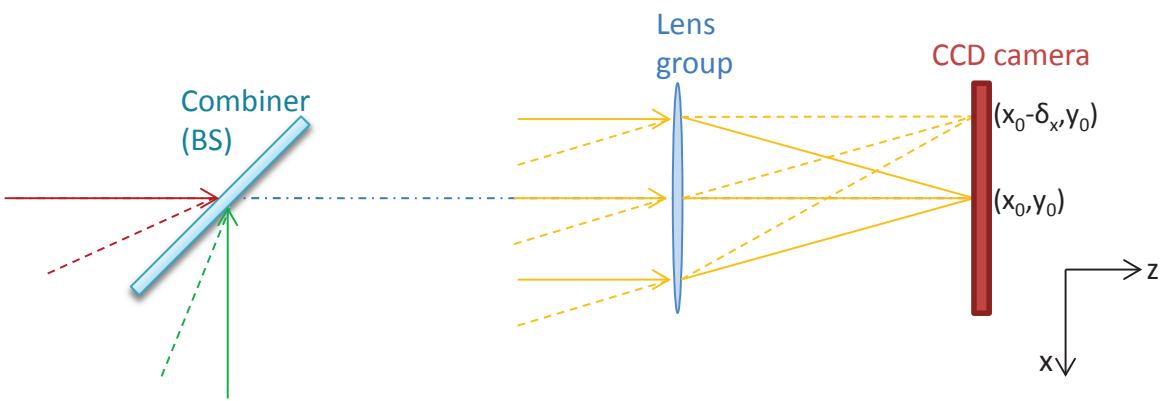

Figure 2. Light rays from a source located on the optical axis of the collimating mirror (solid lines), after passing along opposite arms of the interferometer, reach the beam combiner at right angles. A lens images those rays onto pixel $\left(x_{0}, y_{0}\right)$. As the optical delay line is scanned the pixels record an interferogram. Light rays from an off-axis source (dashed lines) strike the beam combiner symmetrically but at an acute or obtuse angle. These rays are imaged onto pixel $\left(x_{0}-\delta_{x}, y_{0}\right)$, which records an interferogram whose ZPD is displaced $\delta_{x}$ relative to the ZPD for an on-axis source. (Figure adapted from Leisawitz et al. 2003)

by the antennas (or collector mirrors), and we define this as the ' $x$ ' direction, then light from the off-axis source will be brought to a focus on a pixel $\left(x_{0}-\delta_{x}, y_{0}\right)$ with

$$
\delta_{x}=f \theta
$$

and where $f$ is the focal length of the re-imaging lens. In other words the re-imaging lens is performing an optical Fourier transform of the input plane waves. The signal recorded as a function of time by a camera pixel located at $\left(x_{0}-\delta_{x}, y_{0}\right)$ will be an interferogram whose Fourier transform is the spectrum of the off-axis source at the spatial frequency sampled by the baseline $b=b_{0} \cos (\theta)$ which is the projection of the source on the baseline and the zero path difference (ZPD) will be displaced relative to its location for the on-axis source by an amount $d_{Z P D}=b_{0} \sin \theta$. Therefore, by stroking the delay line through a distance greater than that required for spectroscopy, one can allow for the ZPD shift and obtain useful interferometric data for off-axis sources.

Figure 3 shows a schematic of the WIIT experimental setup. Light from the test scene, generated by the Calibrated Hyperspectral Image Projector ${ }^{13}$ (CHIP) and located at the focus of the collimating mirror, is projected into the interferometer. The two collector mirrors feed the two arms of the interferometer. One of these arms consists solely of fixed flat mirrors (fixed arm), while the other includes a pair of mirrors mounted on the delay line stage in a rooftop configuration (delay arm). The delay line scans a range of optical path differences between the two arms of the interferometer. The beams from the two arms are recombined at the beamsplitter, and the output from one of the two output ports is focused onto a CCD camera by a lens group. The source scene is rotated via software, providing access to all possible baseline orientations. The collector mirrors can be moved along a straight rail to vary baseline length. Compared to the testbed set in Cardiff University, WIIT moves both collector mirrors instead of just one arm.

\subsection{CHIP: Calibrated Hyperspectral Image Projector}

CHIP utilizes two Digital Light Processing (DLP) projectors to create custom, spectrally diverse and spatially complex scenes. The two DLP engines (a spectral engine and a spatial engine) operate in series to produce the scene to be measured. First, a broadband source is dispersed onto the digital micromirror device (DMD) of the spectral engine such that individual columns of the mirror are mapped to individual wavelengths. By turning the mirrors in a single column on, the user includes that specific wavelength in the output. The number 


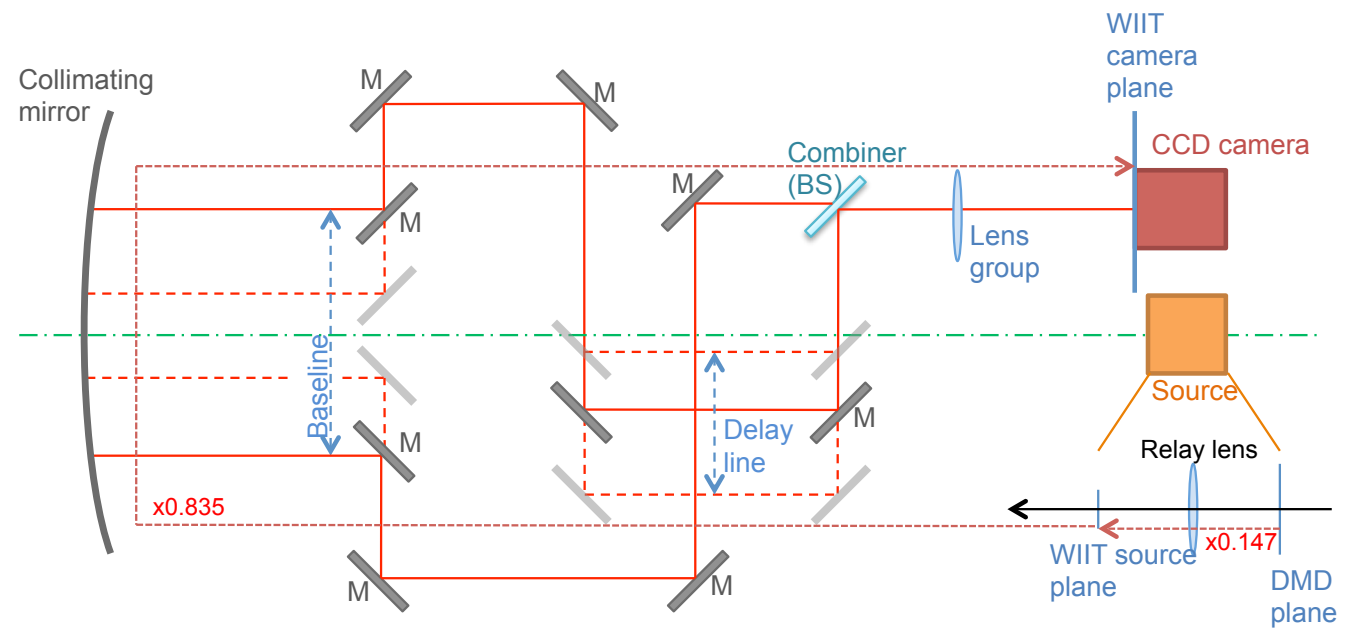

Figure 3. Schematic of the WIIT experimental setup. Light from the test scene (source) located at the focus of the collimating mirror is projected into the interferometer. The two collector mirrors separated by the baseline length feed the two arms of the interferometer. One of these arms consists solely of fixed flat mirrors (lower arm, fixed), while the other includes a pair of mirrors mounted on the delay line stage in a rooftop configuration (upper arm, delay arm). The delay line scans a range of optical path difference between the two arms of the interferometer. The beams from the two arms are recombined at the combiner (beam splitter), and the output from one of the two output ports is focused onto a CCD camera by a lens group. The demagnification of the DMD plane is also shown: a relay lens system images the DMD plane to the WIIT source plane while demagnifying the DMD by a factor 0.147 . The testbed provides an additional demagnification of 0.835 due to the WIIT collimator and lens system.

of on mirrors in a column determines the relative intensity of the spectral component. By selecting numerous columns and appropriately weighting the various spectral components, the user generates a basis spectrum of the hyperspectral scene to be projected. CHIP is capable of producing arbitrary spectra in the band between $420 \mathrm{~nm}$ and $780 \mathrm{~nm}$ with a spectral resolution of up to $5 \mathrm{~nm}$.

The results presented here are based on observations of an astronomically realistic test scene, specifically a simulated far-infrared deep field observation of the extragalactic sky. The original simulation is a hyperspectral cube covering a 1.2 arcmin patch of sky in which the spatial pixels each subtend 0.07 arcsec and the spectral channels span the wavelength range 25 to $400 \mu \mathrm{m}$ in $1 \mu \mathrm{m}$ intervals. The simulated sky contains spatially uniform zodiacal emission calibrated to the COBE DIRBE zodiacal emission model; Galactic cirrus emission was normalized to IRAS observations at $>1$ arcmin angular scales, and extrapolated to finer scales using a power law distribution; and galaxies were distributed in redshift and luminosity according to distribution functions calibrated before the Herschel mission. Each galaxy has a standard morphology and spectral template that includes both continuum and spectral lines.

To project this far-infrared scene for observation with WIIT, the original hyperspectral datacube was regridded to the CHIP resolution, a $280 \times 280$ CHIP pixel sub-field was selected, and the simulated far-IR wavelengths were mapped into the visible wavelength range accessible to CHIP. Apart from this transformation, we aimed for a high- fidelity representation of the scene. We found that nearly all of the spatial and spectral information could be preserved if the field was divided into four wavelength bins, and 8 basis spectra and corresponding basis images were used to enable time-efficient projection with the CHIP. The basis spectra and images were obtained through nonnegative matrix factorization ${ }^{14}$ of the hyperspectral image that had been cropped and divided into four wavelength bins, where we also incorporated calibration data from CHIP into the factorization 

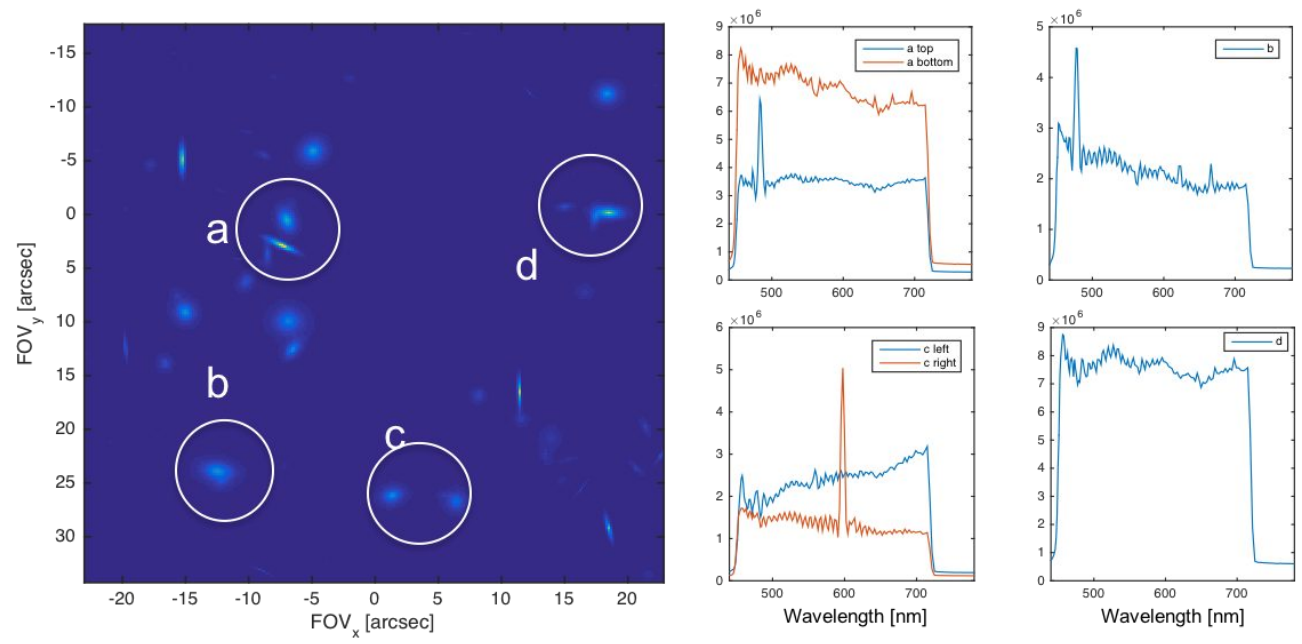

Figure 4. From a simulated hyperspectral far-IR extragalactic deep field, we broke the datacube into four wavelength bins, mapped the far-IR wavelengths into the visible range for projection with CHIP, and used 8 eigenspectra and corresponding eigenimages to capture most of the original spatial and spectral content while enabling time-efficient projection with the CHIP. Right: CHIP projected spectra (blend of eigenspectra) for the bright sources contained in the regions outlined, showing the presence of continuum (zodiacal, cirrus, and galactic) and spectral line emission (from the galaxies at their respective redshifts) from the original far-IR spectral range 307-400 $\mu \mathrm{m}$. Left: A wavelength-integrated image representing the projected 280 x 280-pixel test scene. Four separate WIIT data sets were collected, one for each of the four wavelength bins, but only one of the four is represented here.

algorithm. Fig. 4 illustrates the richness of the hyperspectral scene corresponding to the original $307 \mu \mathrm{m}$ to 400 $\mu \mathrm{m}$ wavelength bin.

The original scene is further surrounded by a set of reference sources (not shown in Fig. 4, which are used in the image synthesis and reconstruction to register the datacubes measured at each baseline rotation to a common baseline orientation. For the reference sources, our goal is to have as much power being emitted by CHIP as possible, and this can be achieved by configuring CHIP so all the mirrors are 'on' spectrally.

\subsection{Data Acquisition with WIIT}

Raw data from the WIIT consists of a series of CCD camera frames corresponding to the sampled optical path lengths and baselines and ancillary data. ${ }^{4,15}$ To facilitate data reduction and analysis the science data and synchronously collected ancillary data ${ }^{15}$ (such as environmental, optical and electro-mechanical metrology) are recorded in the astronomical standard FITS (Flexible Image Transport System) format. All of the data from a single delay line scan and fixed rotation baseline (length and angle) are recorded in a single FITS file. The header and housekeeping data records in the file include all the information needed to calculate the baseline vector.

At a given baseline position and rotation a datacube is collected. Each plane of the cube is a 90x90 pixel image if the full FOV is recorded. Each cube contains a number of planes or frames proportional to the total optical path range to be scanned and the delay line increments, with the finest step size measured with the metrology system being $9.89 \mathrm{~nm}$. For example, if the desired optical path range is $60 \mu m$ this corresponds to approximately 6000 steps of the delay line and thus this number of frames to each baseline cube.

Spatially, a minimum baseline of $36 \mathrm{~mm}$ and a maximum baseline of $226 \mathrm{~mm}$ is set, with a baseline increment of $10 \mathrm{~mm}$. The interferometer plane rotates with respect to the input scene such that the the arc length between consecutive rotations for a given baseline length remains constant, providing a grid-style sampling of the uv-map. 

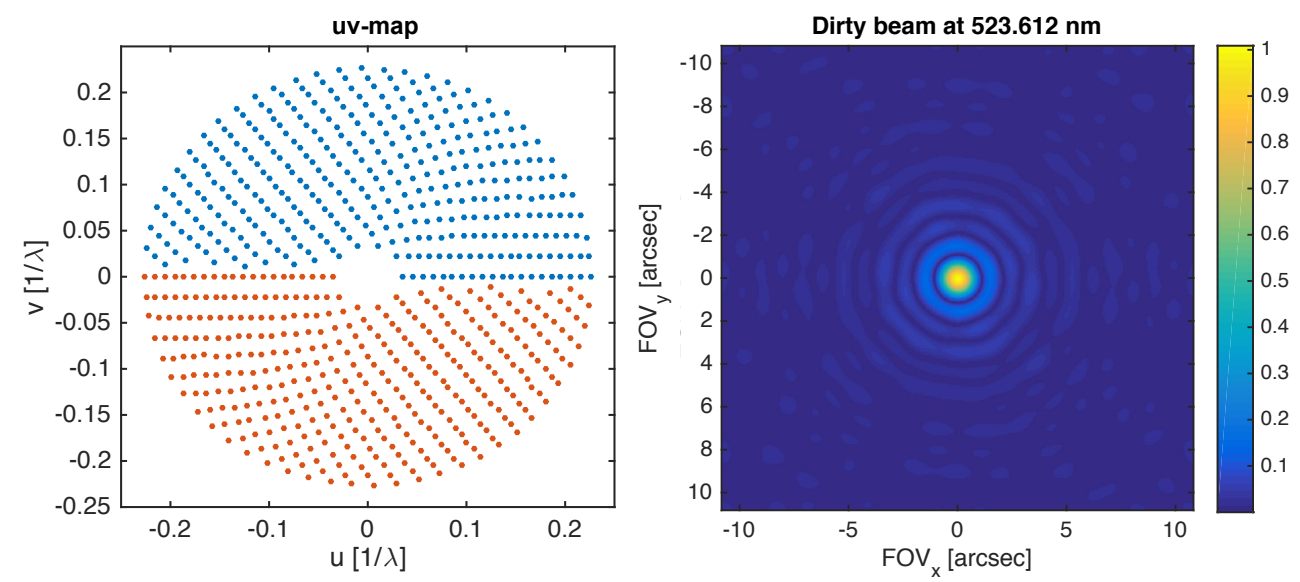

Figure 5. Left: uv-coverage, where blue indicates the sampled uv positions and red its negative counterpart. Right: Normalized interferometric dirty beam at 523.6nm.

Fig.5 (left) shows the sampling of the uv-map. The corresponding interferometric dirty beam at $523.6 \mathrm{~nm}$ is shown in Fig. 5 (right). It can be observed that the dirty beam pattern is similar to the Airy pattern at the centre of the field of view, but presents some distortion with a power level comparable to that of the secondary lobe of the dirty beam. This distortion is due to the fact that the sampling of the uv-map is not completely uniform.

Regarding the optical system, the entrance pupil of WIIT comprises the two apertures of $25 \mathrm{~mm}$ diameter that constitute the interferometric baseline. The optical system that projects the light on the CCD camera has an $\mathrm{F} / \#$ of 80 , and the CCD pixel size is $\Delta \mathrm{x}=16 \mu \mathrm{m}$, corresponding to the camera focal length of $2 \mathrm{~m}$. With these parameters one can define the focal plate scale (FPS) as FPS $=\Delta x / f=1.6 \operatorname{arcsec} /$ pixel. Hence, the Airy pattern is oversampled with respect to the Nyquist sampling by a factor of $\sim 2.5$ to 4.8 at $420 \mathrm{~nm}$ to $780 \mathrm{~nm}$, respectively.

We used the simulator FIInS ${ }^{16,17}$ to generate a set of FITS files, more specifically 364 files (one per baseline angle and rotation), each $38.2 \mathrm{MB}$, that contain the simulated datacube. Fig. 6 (left) shows the integrated intensity for the current simulation with a $36 \mathrm{~mm}$ baseline length. In the science region (within the red box) it can be observed that the sources in regions a, $\mathrm{c}$ and $\mathrm{d}$ are not resolved spatially, as expected, because the footprint of a single camera pixel is bigger than the angular separation of these sources. Fig. 6 (right) shows representative interferograms at regions a, b, c and d for a $46 \mathrm{~mm}$ baseline (top) and a $226 \mathrm{~mm}$ baseline (bottom) at 0 degrees rotation. Focusing in region a, it can be observed that with the longer baseline one can resolve the 2 sources within that region, as longer baselines induce a bigger shift of the centre of the fringe packet (ZPD) of each interferogram, given by $d_{Z P D}=b \sin \theta$.

From the simulation one can also observe the strong effect the emitted total intensity per source has in the amplitude of the recorded interferogram, i.e. in region a, source a-top emits twice as much energy as source a-bottom (see Fig. 4 right), hence the amplitude of the interferogram corresponding to the source a-top doubles the amplitude of the interferogram corresponding to the source a-bottom. 

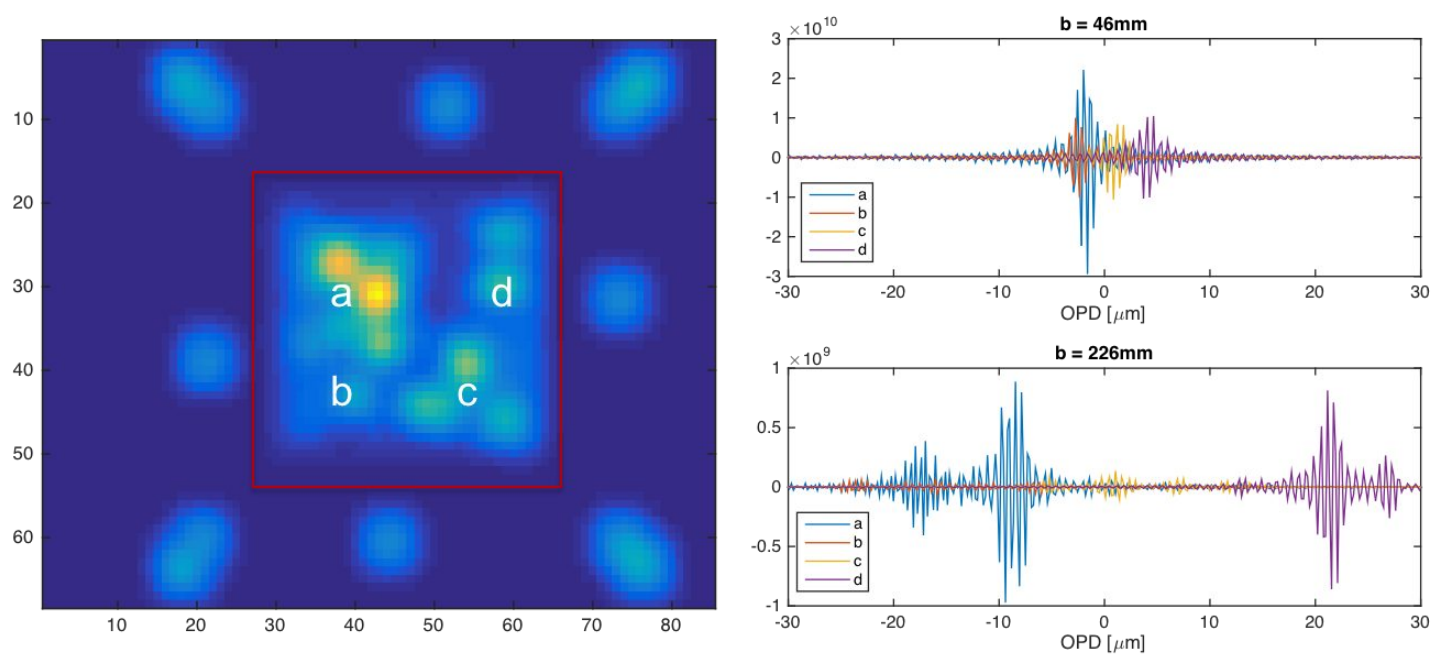

Figure 6. Left: Simulated integrated intensity of a WIIT measurement at the camera plane. The red box indicates the science region. The sources outside the red box are the reference sources. Right: interferograms at science regions a, b, $\mathrm{c}$ and $\mathrm{d}$ for a $46 \mathrm{~mm}$ baseline (top) and a $226 \mathrm{~mm}$ baseline (bottom) at 0 degrees rotation.

\section{RECONSTRUCTION OF THE INPUT SCENE}

Once the interferograms have been acquired the next step is to extract the spatial and spectral information for each pixel of the datacube. Because each pixel on the detector plane acts as a single double Fourier interferometer, the single pixel data processing can be applied to the full array as a set of individual pixels.

The reconstruction of the WIIT scene from the recorded interferograms is a two-step process: a spectral inverse Fourier transform, and a spatial inverse Fourier transform. For a given camera pixel (v,h), the 'dirty' spectra $S_{v, h}\left(\nu, b_{j}\right)$ are extracted from the set of interferograms $I_{v, h}\left(\delta, b_{j}\right)$ for each baseline using Eq. 2:

$$
S_{v, h}\left(\nu, b_{j}\right)=\sum_{i}^{N} I_{v, h}\left(\delta_{i}, b_{j}\right) \exp \left(-i 2 \pi \nu \delta_{i}\right) d \delta
$$

The next step is the extraction of the spatial features of $S_{v, h}$; this is the dirty datacube, $S k y_{v, h}$. This step converts a single pixel from each baseline into a datacube covering the angle subtended by the FWHM of the telescope beam (or entrance pupil Airy pattern). To extract the spatial features, one has to perform the twodimensional Fourier transform of the uv-map. Combining each baseline position $b_{j}$ and each wavenumber $\nu_{k}$, the dirty datacube is calculated as

$$
\left.S k y_{v, h}\left(\theta_{x} \theta_{y} ; \nu_{k}\right)=\sum_{j=1}^{N b} \mid \operatorname{Im}\left\{S_{v, h}\left(\nu_{k}, b_{j}\right)\right\} \cos \left(2 \pi\left(u_{j, k} \theta_{x}+v_{j, k} \theta_{y}\right)\right)-\operatorname{Re}\left\{S_{v, h}\left(\nu_{k}, b_{j}\right)\right\} \sin \left(2 \pi\left(u_{j, k} \theta_{x}+v_{j, k} \theta_{y}\right)\right)\right\} \mid
$$

where $u_{j, k}=b_{x, j} \nu_{k}$ and $v_{j, k}=b_{y, j} \nu_{k}$ are the spatial frequencies. In this operation, prior knowledge of the sky grid $\left(\theta_{x}, \theta_{y}\right)$ has to be used to mosaic the individual $S k y_{v, h}$ datacubes and reconstruct the wide-field scene.

Fig. 7 (left) shows the spatial distribution of the reconstructed scene or dirty image of the science region. Spatially, all the sources are resolved, but one can observe the distortions induced by the dirty beam, with sidelobe power comparable to that of the dimmer sources. By applying post-processing algorithms such as CLEAN $^{18}$ and MEM, ${ }^{19}$ these artifacts will be minimized. 

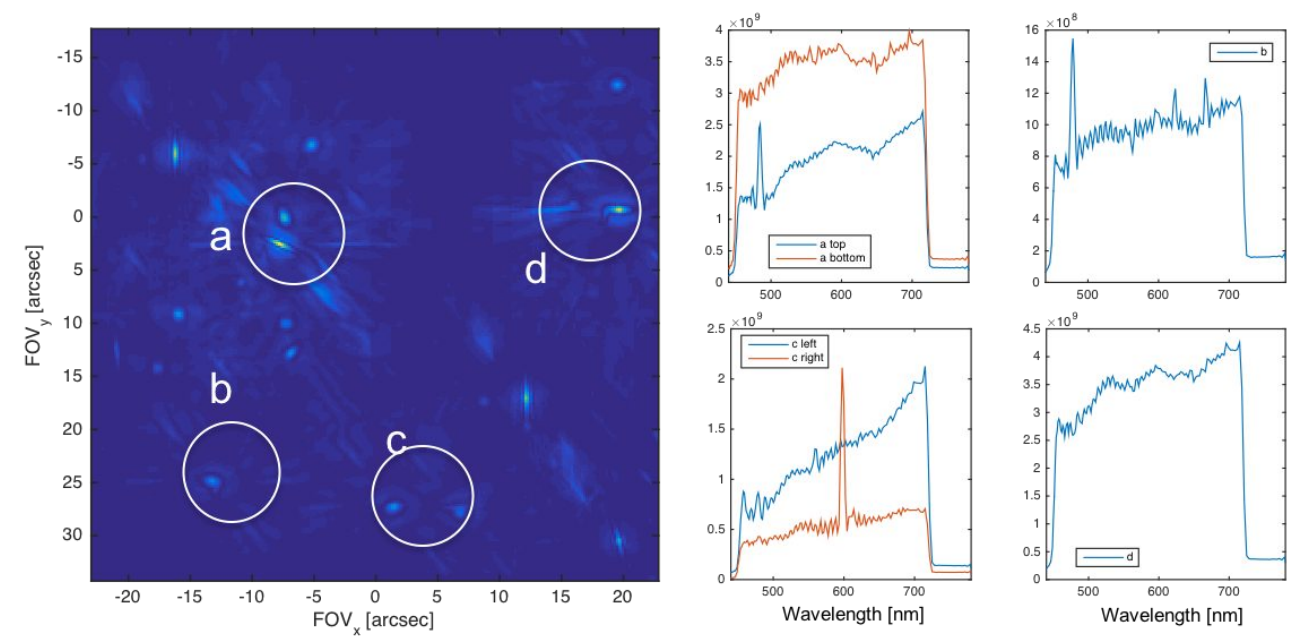

Figure 7. Spatial structure of the reconstructed scene or dirty image. Left: spectrally integrated intensity of the full science scene. Right: derived spectra of each source in the science regions a, b, c and d.

The derived spectra are in good but not perfect agreement with the known input spectra, as shown in Fig. 7 (right). In the cases where one of the extended sources is substantially more intense than its companion, there is a transfer of power from the brighter to the fainter source at longer wavelengths. This is due to the fact that the width of the interferometric dirty beam is bigger at longer wavelengths.

\section{PROCESSING OF WIIT DATA}

Although WIIT resides in the well-controlled environment of the AIM Lab, there are aspects of the interferometer that require the data to be preprocessed prior to employing image synthesis techniques. For example, there is heat generated by WIIT's imaging camera and CHIP that cause mild motion between image measurements for a single baseline length and orientation. Also, the lamp that provides the illumination for CHIP fluctuates in its overall intensity during each delay line scan, causing features in the collected data that appear as low-order signatures for the fringes at each pixel. Due to the micromirror devices that control how the hyperspectral projector operates, CHIP also imparts a spatially variant background on the scenes being measured. WIIT's CCD also has hot pixels, typically comprising the bottom few rows of pixels in the image frame, that must be removed in processing. Finally, because baseline orientations are varied by digitally rotating the scene being projected by CHIP, all baseline measurements must be registered and resampled to the same orientation prior to image synthesis. To aid in the preprocessing of the data, a collection of image measurements are taken to identify the background imparted by CHIP and the imaging camera before starting each delay line scan.

The procedure for preprocessing WIIT datasets starts by processing each datacube, which consists of all measurements for a delay line scan for each baseline length and angle, independently. First, the fixed background created by the hot pixels of WIIT's imaging camera are removed from all background and data measurements. The remaining background frames registered such that all background frames no longer suffer from image shifts caused by the mild turbulence in the system before averaging the background frames together. The data frames for each datacube are also registered to the first measured frame of the delay line scan. Before removing the background caused by CHIP from all of the data frames, we first remove the intensity fluctuations caused by CHIP's illumination source. This is accomplished by using a high-pass filter designed with the spectral bandpass 
of CHIP in mind, which leaves us with the desired fringes while removing the low-order signatures cause by the lamp fluctuations. The averaged background image is then registered to the average of the data frames prior to removing the background from all data frames in the datacube. Fig. 8 shows the result of the preprocessing of one datacube, corresponding to a baseline length of $46 \mathrm{~mm}$ and 0 degree orientation. As in the simulation, we can observe how the increase in baseline length shifts the ZPD of each interferogram, informing us about the spatial features within the scene. Our choice of presenting the $96 \mathrm{~mm}$ baseline is due to the fact that for longer baselines the interferogram amplitude is comparable to the noise level and it is difficult to identify the corresponding ZPD.
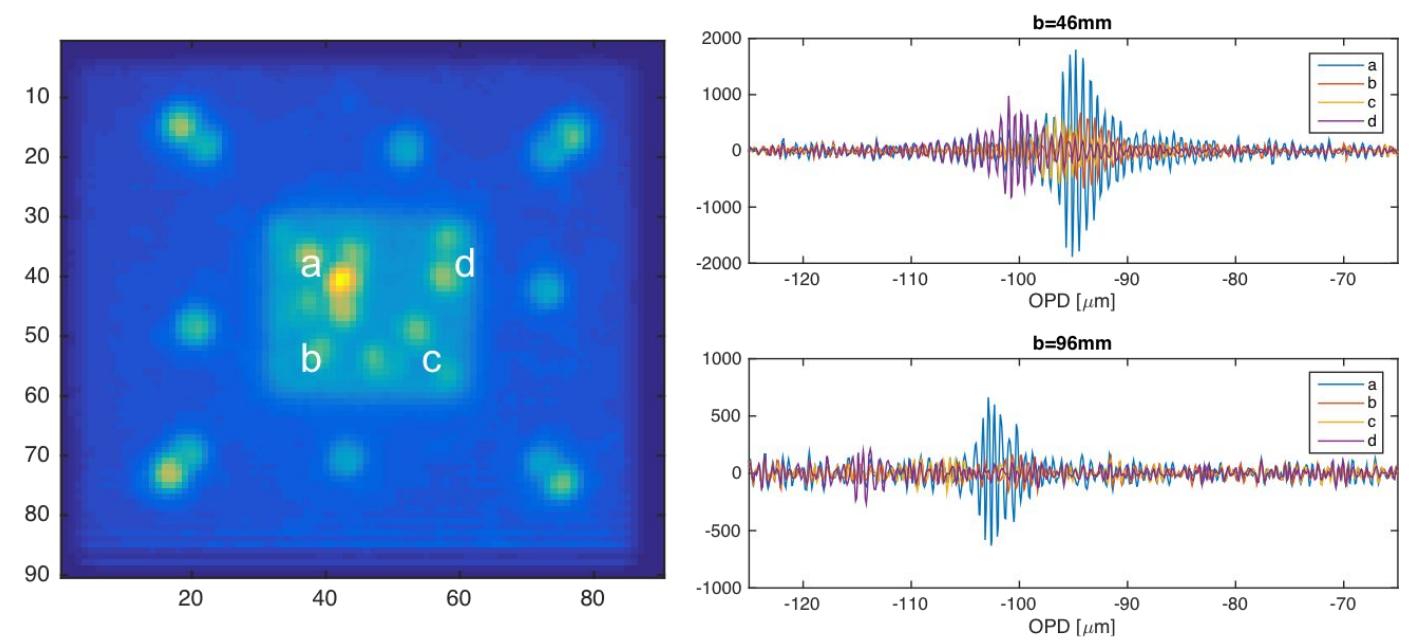

Figure 8. Left: integrated intensity of a WIIT measurement at the camera plane, for a $46 \mathrm{~mm}$ baseline length and 0 degree rotation, where we can observe both the science region and the reference sources. Right: interferograms at science regions $\mathrm{a}, \mathrm{b}, \mathrm{c}$ and $\mathrm{d}$ for a $46 \mathrm{~mm}$ baseline (top) and a $96 \mathrm{~mm}$ baseline (bottom) at 0 degrees rotation.

Once each datacube has been processed independently, registration of the entire dataset is possible. First, we take the average data frame from each datacube. We then use a chirp z-transform image registration approach ${ }^{20}$ to register all the images to the image from the first datacube in the dataset. The rotation angle between measurements is known very well because source rotation is performed digitally prior to image projection, but there are remaining image translations that must be recovered. The point spread function of WIIT's imaging camera is asymmetric, so a deconvolution step is added to the image registration procedure to improve the accuracy of the retrieved translation results. The chirp z-transform algorithm is then used to orient all data frames based on the results of the image registration algorithm. Fig. 9 shows the results of the registration of the $46 \mathrm{~mm}$ datacubes to the first datacube (0 degrees rotation).

Once the data is registered to a common datacube, further steps have to be performed before we can synthesize the full scene. For example, for a given baseline length, one has to find the center of rotation to further align the interferograms to a common optical path difference. To perform this step, we find the ZPD of the reference sources in each datacube and fit a plane. The intersection of the planes corresponding to all the rotations for a given baseline length gives us the center of rotation. We are currently working in the development of the algorithm to align all datacubes for all the baseline lengths and rotations to the common OPD that will allow to finalize the synthesis of double Fourier modulation data. 

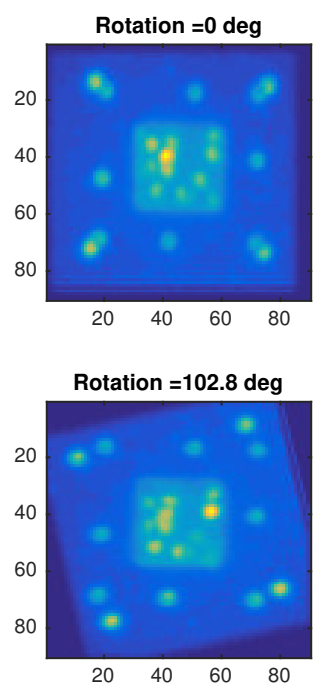
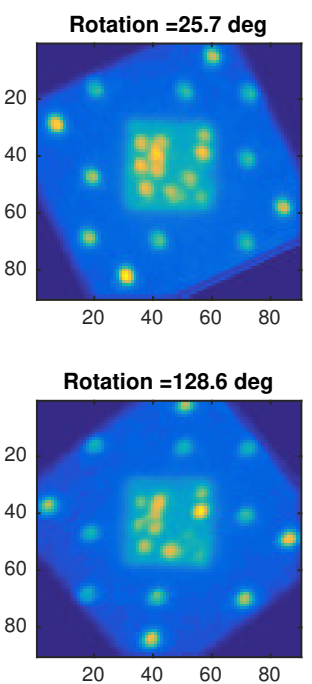
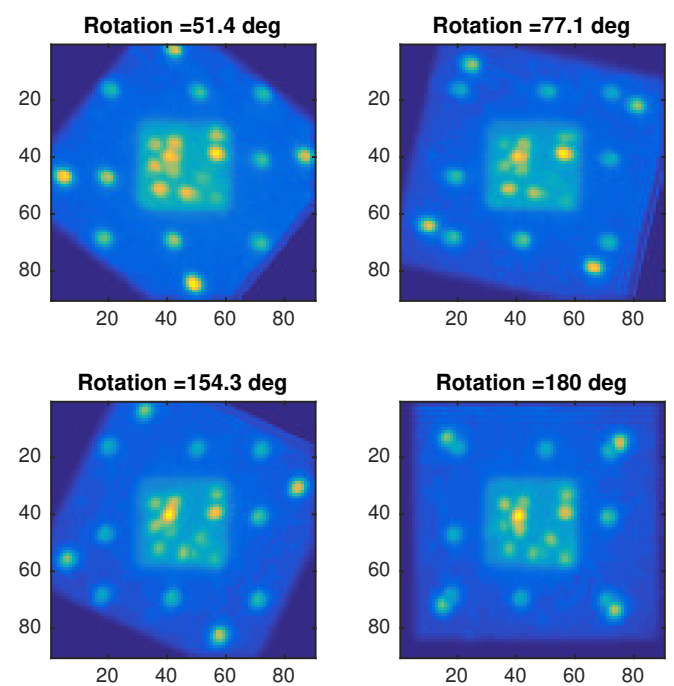

Figure 9. Integrated intensity of WIIT measurements after the registration of the $46 \mathrm{~mm}$ datacubes to the first datacube (0 degrees rotation). It can be observed the presence of the reference sources used for the registration of the cubes, as they are stationary in each cube.

\section{CONCLUSIONS AND FUTURE WORK}

Double Fourier modulation is a powerful technique that will allow future missions to perform simultaneous spectral and spatial measurements of astronomical sources at high angular resolution. Although the future of space interferometry is uncertain, it will eventually become the only option to access astrophysics that require very high angular resolution. The novel aspect of WIIT, which is the extension of the field of view, opens a realm of possibilities as it allows the systematic measurement of numerous sources within the FOV as well as direct imaging. The simulations presented here show how we can reconstruct complex scenes with this technique. We are currently working on the development of new algorithms that will allow the full reconstruction of scenes from DFM experimental data. Although at present we are able to extract spatial information from interferograms, we aim to develop a fully automated software to perform the synthesis of a full set of DFM data from our WIIT experiments.

\section{ACKNOWLEDGMENTS}

This research was supported by an appointment of the lead author to the NASA Postdoctoral Program at the Goddard Space Flight Center, administered by USRA through a contract with NASA.

\section{REFERENCES}

[1] Kouveliotou, C., Agol, E., Batalha, N., Bean, J., Bentz, M., Cornish, N., Dressler, A., Figueroa-Feliciano, E., Gaudi, S., Guyon, O., et al., "Enduring quests-daring visions (nasa astrophysics in the next three decades)," arXiv preprint arXiv:1401.3741 (2014). 
[2] Itoh, K. and Ohtsuka, Y., "Fourier-transform spectral imaging: retrieval of source information from threedimensional spatial coherence," J. Opt. Soc. Am. A 3, 94-100 (Jan 1986).

[3] Mariotti, J. and Ridgway, S. T., "Double Fourier spatio-spectral interferometry - Combining high spectral and high spatial resolution in the near infrared," Astron. Astrophys. 195, 350-363 (Apr. 1988).

[4] Leisawitz, D. T., Frey, B. J., Leviton, D. B., Martino, A. J., Maynard, W. L., Mundy, L. G., Rinehart, S. A., Teng, S. H., and Zhang, X., "Wide-field imaging interferometry testbed I: purpose, testbed design, data, and synthesis algorithms," in [SPIE Conference Series], Shao, M., ed., 4852, 255-267 (Feb. 2003).

[5] Ohta, I. S., Hattori, M., and Matsuo, H., "Development of a multi-Fourier-transform interferometer: fundamentals," Appl. Opt. 45, 2576-2585 (Apr. 2006).

[6] Ohta, I. S., Hattori, M., and Matsuo, H., "Development of a multi-Fourier-transform interferometer: imaging experiments in millimeter and submillimeter wave bands," Appl. Opt. 46, 2881-2892 (May 2007).

[7] Grainger, W. F., Juanola-Parramon, R., Ade, P. A. R., Griffin, M., Liggins, F., Pascale, E., Savini, G., and Swinyard, B., "Demonstration of spectral and spatial interferometry at THz frequencies," Appl. Opt. 51, 2202-2211 (Apr 2012).

[8] Rinehart, S. A., Leisawitz, D. T., Bolcar, M. R., Chaprnka, K. M., Lyon, R. G., Maher, S. F., Memarsadeghi, N., Sinukoff, E. J., and Teichman, E., "Recent progress in wide-field imaging interferometry," in [Society of Photo-Optical Instrumentation Engineers (SPIE) Conference Series], Society of Photo-Optical Instrumentation Engineers (SPIE) Conference Series 7734 (July 2010).

[9] Leisawitz, D., Baker, C., Barger, A., Benford, D., Blain, A., Boyle, R., Broderick, R., Budinoff, J., Carpenter, J., Caverly, R., Chen, P., Cooley, S., Cottingham, C., Crooke, J., Dipietro, D., Dipirro, M., Femiano, M., Ferrer, A., Fischer, J., Gardner, J. P., Hallock, L., Harris, K., Hartman, K., Harwit, M., Hillenbrand, L., Hyde, T., Jones, D., Kellogg, J., Kogut, A., Kuchner, M., Lawson, B., Lecha, J., Lecha, M., Mainzer, A., Mannion, J., Martino, A., Mason, P., Mather, J., McDonald, G., Mills, R., Mundy, L., Ollendorf, S., Pellicciotti, J., Quinn, D., Rhee, K., Rinehart, S., Sauerwine, T., Silverberg, R., Smith, T., Stacey, G., Stahl, H. P., Staguhn, J., Tompkins, S., Tveekrem, J., Wall, S., and Wilson, M., "The space infrared interferometric telescope (SPIRIT): High-resolution imaging and spectroscopy in the far-infrared," Advances in Space Research 40, 689-703 (2007).

[10] Leisawitz, D. T., Bolcar, M. R., Lyon, R. G., Maher, S. F., Memarsadeghi, N., Rinehart, S. A., and Sinukoff, E. J., "Developing wide-field spatio-spectral interferometry for far-infrared space applications," in [Society of Photo-Optical Instrumentation Engineers (SPIE) Conference Series], Society of Photo-Optical Instrumentation Engineers (SPIE) Conference Series 8445 (2012).

[11] Lyon, R. G., Rinehart, S. A., Leisawitz, D. T., and Memarsadeghi, N., "Wide-field imaging interferometry testbed (WIIT): image construction algorithms," in [Society of Photo-Optical Instrumentation Engineers (SPIE) Conference Series], Society of Photo-Optical Instrumentation Engineers (SPIE) Conference Series 7013 (July 2008).

[12] Iacchetta, A. S. and Fienup, J. R., "Wide-field spatiospectral interferometry: theory and imaging properties," J. Opt. Soc. Am. A 34, 1896-1907 (Oct 2017).

[13] Bolcar, M. R., Leisawitz, D. T., Maher, S. F., and Rinehart, S. A., "Demonstration of the Wide-field Imaging Interferometer Testbed using a Calibrated Hyperspectral Image Projector," in [Society of PhotoOptical Instrumentation Engineers (SPIE) Conference Series], Society of Photo-Optical Instrumentation Engineers (SPIE) Conference Series 8445 (2012). 
[14] Iacchetta, A. S., Fienup, J. R., Leisawitz, D. T., and Bolcar, M. R., "Nonnegative matrix factorization for efficient hyperspectral image projection," in [Imaging Spectrometry XX], 9611, 96110Y, International Society for Optics and Photonics (2015).

[15] Leviton, D. B., Frey, B. J., Leisawitz, D. T., Martino, A. J., Maynard, W. L., Mundy, L. G., Rinehart, S. A., Teng, S. H., and Zhang, X., "Wide-field imaging interferometry testbed 3: metrology subsystem," in [Society of Photo-Optical Instrumentation Engineers (SPIE) Conference Series], Shao, M., ed., Society of Photo-Optical Instrumentation Engineers (SPIE) Conference Series 4852, 827-838 (Feb. 2003).

[16] Juanola-Parramon, R., Leisawitz, D. T., Bolcar, M. R., Maher, S. F., Rinehart, S. A., Iacchetta, A., and Savini, G., "The wide-field imaging interferometry testbed (wiit): recent progress in the simulation and synthesis of wiit data," in [Optical and Infrared Interferometry and Imaging V], 9907, 990740, International Society for Optics and Photonics (2016).

[17] Juanola-Parramon, R., Fenech, D., and Savini, G., "Architecture and performance of the space-based farinfrared interferometer instrument simulator," Monthly Notices of the Royal Astronomical Society 457(4), 3457-3469 (2016).

[18] Högbom, J., "Aperture synthesis with a non-regular distribution of interferometer baselines," Astron. Astrophys. Suppl 15(1974), 417-426 (1974).

[19] Gull, S. and Skilling, J., "Maximum entropy method in image processing," Communications, Radar and Signal Processing, IEE Proceedings F 131(6), 646-659 (1984).

[20] Alexander S. Iacchetta, J. R. F. and Leisawitz, D. T., "Rotation and translation registration of bandlimited interferometric images using a chirp z-transform," in [Proc. SPIE], Society of Photo-Optical Instrumentation Engineers (SPIE) Conference Series 9907 (2016). 